\title{
Efficacy and Safety of Lipase Inhibitor Orlistat in Japanese with Excessive Visceral Fat Accumulation: 24-Week, Double-Blind, Randomized, Placebo- Controlled Study
}

\author{
Kohji Shirai · Toru Fujita · Michitaka Tanaka · Yuka Fujii • \\ Masatsugu Shimomasuda · Soichi Sakai · Yoshishige Samukawa
}

Received: September 3, 2018 / Published online: December 10, 2018

(C) The Author(s) 2018

\begin{abstract}
Introduction: Orlistat is an inhibitor of pancreatic lipase and is used as an anti-obesity drug in many countries. However, there are no data available regarding the effects of orlistat on visceral fat accumulation in Japanese subjects. Therefore, this comparative, placebo-controlled, double-blind, randomized study aimed to evaluate the efficacy and safety of orlistat in Japanese participants with excessive visceral fat accumulation and without dyslipidemia, diabetes mellitus, and hypertension ("metabolic diseases").

Methods: The study population included Japanese participants with excessive visceral fat accumulation (waist circumference $\geq 85 \mathrm{~cm}$ in males and $\geq 90 \mathrm{~cm}$ in females, which
\end{abstract}

Enhanced Digital Features To view enhanced digital features for this article go to https://doi.org/10.6084/ m9.figshare.7297526.

Electronic Supplementary Material The online version of this article (https://doi.org/10.1007/s12325018-0835-5) contains supplementary material, which is available to authorized users.

K. Shirai

Department of Internal Medicine, Mihama Hospital, Chiba, Japan

T. Fujita $(\bowtie) \cdot$ M. Tanaka · Y. Fujii .

M. Shimomasuda · S. Sakai - Y. Samukawa

Taisho Pharmaceutical Co., Ltd., Tokyo, Japan

e-mail: too-fujita@taisho.co.jp corresponds to a visceral fat area of $100 \mathrm{~cm}^{2}$ ) and without metabolic diseases. Following a 12-week observation term, participants were randomized to the orlistat $60 \mathrm{mg}$ group $(n=100)$ or placebo group $(n=100)$. Both drugs were administered orally three times daily for 24 weeks. Participants were also counseled to improve their diet and to maintain exercise throughout the study. Visceral fat area, subcutaneous fat area, waist circumference, body weight, body mass index, adverse reactions, laboratory tests, and blood pressure were regularly assessed.

Results: Visceral fat area, waist circumference, and body weight were significantly reduced in the orlistat group (mean \pm standard error, $-13.50 \pm 1.52 \%,-2.51 \pm 0.25 \%$, and -2.79 $\pm 0.30 \%$, respectively) compared to the placebo group $(-5.45 \pm 1.50 \%,-1.55 \pm 0.26 \%$, and $-1.22 \pm 0.28 \%$, respectively) at the last assessment. The main adverse reactions were defecation-related symptoms including oily spotting and flatus with discharge, resulting from the pharmacological effects of orlistat. Most adverse reactions were mild, and none were serious or severe.

Conclusion: Orlistat administration reduced visceral fat area, waist circumference, and body weight in Japanese participants with excessive visceral fat and without metabolic diseases. In addition, safety was confirmed with a tolerable profile. Orlistat may be useful to reduce 
excessive visceral fat accumulation when used in combination with diet and exercise.

Trial Registration: Japan Pharmaceutical Information Center identifier, JapicCTI-184005.

Funding: Taisho Pharmaceutical Co., Ltd.

Keywords: Body weight; Double-blind; Efficacy; Japanese; Lipase inhibitor; Obesity; Orlistat; Placebo-controlled; Randomized; Safety; Visceral fat; Waist circumference

\section{INTRODUCTION}

Obesity is frequently associated with metabolic disorders such as dyslipidemia, diabetes mellitus, and hypertension, and causes coronary artery disease, cerebrovascular disease, sleep apnea, renal disorders, bone/joint disorders, menstrual disorders, and other health problems $[1,2]$. The Organisation for Economic Co-operation and Development has ranked obesity as the greatest threat to public health worldwide, and issued a warning related to medical expenditures for the treatment of obesity-related diseases globally [3]. The incidence of obesity-related dyslipidemia, diabetes mellitus, and hypertension as well as obesity-related cardiovascular diseases has indeed been increasing worldwide. Various indices of obesity have been proposed; for example, body weight, body mass index (BMI), waist circumference, and body fat. In the USA and Europe, BMI is widely used as an index for the diagnosis and treatment of obesity $[4,5]$.

In Japan, despite a lower grade of obesity in comparison to the US and European populations, the incidence of dyslipidemia, diabetes mellitus, and hypertension ("metabolic diseases") caused by obesity is high, indicating that metabolic diseases caused by obesity can develop even in people with mild obesity. Furthermore, body fat distribution is known to be correlated with the complications observed in obesity, and visceral fat accumulation is more frequently associated with metabolic diseases caused by obesity (e.g., insulin resistance) compared to subcutaneous fat accumulation [2].
Several clinical studies have demonstrated that the number of obesity-related cardiovascular risk factors increases with an increase in visceral fat accumulation $[2,6]$, suggesting a close relationship between excessive visceral fat and the occurrence and aggravation of metabolic diseases caused by obesity [7, 8]. The mechanism by which visceral fat accumulation is associated with insulin resistance is not yet clearly established, and several hypotheses have been proposed. One mechanism suggests that excessive formation of free fatty acids from visceral fat, whereby fatty acids enter the liver and cause impaired glucose metabolism and insulin resistance, may be responsible. Excessive visceral adipose tissue is believed to secrete tumor necrosis factor- $\alpha$, and this may also lead to the development of insulin resistance and other metabolic diseases caused by obesity [2]. Several studies have reported that reduction in visceral fat accumulation leads to improvement in metabolic diseases caused by obesity $[9,10]$. Therefore, visceral fat accumulation is used as an index for the diagnosis and treatment of obesity in Japan [2].

Management of visceral fat accumulation should start with modifications of daily life habits comprising diet and exercise [2]. Patients with metabolic diseases may need medical treatment, and people with visceral type of obesity without metabolic diseases remain at risk of developing such diseases in the future. Generally, such individuals are required to manage their own health regimens. However, lifestyle improvements to achieve significant body weight loss are often insufficient, and long-term reduction of body weight is difficult to maintain [11-14]. Consequently, development of new, effective, and safe methods to reduce visceral fat accumulation is required for individuals with visceral type of obesity who are at risk of developing metabolic diseases.

Several classes of drugs are currently available for body weight reduction. Among these, one class (drugs such as mazindol and fenfluramine) decreases appetite by inhibiting the central nervous system, while another decreases absorption of glucose and/or fat. Orlistat is a pancreatic lipase inhibitor and inhibits fat absorption in the digestive tract. Because it does 
not affect the central nervous system, orlistat may not have addictive properties.

A clinical study conducted in black and white populations in the USA and Sweden [15] demonstrated that orlistat successfully reduces visceral fat accumulation. Orlistat is now used in more than 120 countries, but not in Japan, and there are no data available regarding the effects of orlistat on visceral fat accumulation in Japanese individuals. Therefore, we conducted a double-blind, randomized, placebo-controlled, comparative, phase III study to evaluate the efficacy and safety of orlistat $60 \mathrm{mg}$ administered three times daily for 24 weeks in Japanese participants with excessive visceral fat accumulation and without metabolic diseases.

\section{METHODS}

\section{Participants}

Japanese participants aged 18 years or older with excessive visceral fat but without metabolic diseases were enrolled in this study. Excessive visceral fat accumulation was defined as a waist circumference of $\geq 85 \mathrm{~cm}$ for males and $\geq 90 \mathrm{~cm}$ for females, which corresponds to a visceral fat area of $100 \mathrm{~cm}^{2}$ [2]. Individuals who were receiving medical treatment for obesity, diabetes mellitus, dyslipidemia, hypertension, hyperuricemia, gout, or fatty liver were excluded. Secondary obesity, individuals with $\mathrm{BMI} \leq 22.0 \mathrm{~kg} / \mathrm{m}^{2}$ (optimal BMI in Japan is defined as $22.0 \mathrm{~kg} / \mathrm{m}^{2}$ [16]), and individuals with $\mathrm{BMI} \geq 35.0 \mathrm{~kg} / \mathrm{m}^{2}$ were excluded. Furthermore, individuals taking cyclosporine, warfarin, amiodarone, or other drugs interfering with the effects of orlistat were also excluded.

\section{Study Design}

On the basis of primary endpoint estimates, standard deviation (SD) estimates, power, and predicted dropout rate projected from preliminary studies, the necessary number of participants was calculated to be 200, with 100 in the orlistat group and 100 in the placebo group. The participants were randomly divided into two groups. The study was performed with a doubleblind design, and a placebo was used for the control drug. The placebo could not be visually differentiated from orlistat. A capsule of placebo or orlistat $60 \mathrm{mg}$ was administered orally three times daily with meals (during or within an hour after breakfast, lunch, and dinner).

The study comprised a 12-week observation term, 24-week treatment term, and 12-week post-observation term. Participants were advised to reduce their daily calorie intake by $200-400 \mathrm{kcal}$, depending on body weight, and to improve their dietary habits throughout the study period. They were also advised not to change their exercise habits significantly throughout the study period. Those who improved their daily life habits and had an increase or sufficient reduction in the waist circumference during the observation term were excluded, and the remaining participants were enrolled in the treatment term. Only those participants with a reduced waist circumference after 24 weeks of treatment compared to baseline entered the post-observation term. Those with an unchanged or increased waist circumference, in addition to participants who withdrew from the study, did not enter the postobservation term.

Body measurements and consultation were performed every 4 weeks. Laboratory testing and blood pressure measurements were performed every 4 weeks during the observation term and treatment term. Glycated hemoglobin (HbA1c) was measured every 12 weeks during the observation term and treatment term. Body measurements consisted of height (only measured at the beginning of the observation term), body weight, and waist circumference. Waist circumference was measured using methods published in the guidelines by the Japan Society for the Study of Obesity [2]. BMI was calculated from body weight and height. Areas of visceral fat and subcutaneous fat were measured using computed tomography (CT) at the beginning of the observation term and treatment term, and at week 12 and week 24 during the treatment term. CT images of visceral and subcutaneous fat at the umbilical level were analyzed using visceral fat area measurement software (Fat Scan, Version 5.0, East Japan Institute of 
Technology Co., Ltd., Ibaraki, Japan). CT was performed using methods published by the Japan Society for the Study of Obesity [2]. Participants were required to fast for at least $10 \mathrm{~h}$ before body measurements, abdominal CT, and laboratory testing.

Adverse events were defined as any unfavorable event or the presence or signs of medically unintended complications from the beginning of treatment to the end of treatment. All adverse events were recorded during physician examinations or by self-reporting by the participants. The investigator determined whether a causal relationship existed between the adverse event and the investigational drug; all events except those which were judged to be "not related" were regarded as adverse reactions.

This study is registered with the Japan Pharmaceutical Information Center (identifier: JapicCTI-184005). The study was implemented in accordance with Good Clinical Practice guidelines and the ethical principles of the Helsinki Declaration of 1964, as revised in 2013, concerning human and animal rights, and Springer's policy concerning informed consent has been followed. This study was approved by the institutional review board of each participating institution. Before participation, an explanation of the study was given to all candidates for participation using the explanatory/ informed consent document, and voluntary written informed consent was obtained from participants with a complete understanding of the content.

\section{Assessments}

The primary efficacy endpoints were the percentage change in visceral fat area from baseline to weeks 12 and 24 of treatment, and the percentage change in waist circumference from baseline to each assessment during the treatment term. The secondary efficacy endpoints were the amount of change in visceral fat area and waist circumference; percentage and amount of change in body weight and BMI; the rates of achievement of a 3\% reduction in waist circumference and a 3\% reduction in body weight (defined as the percentage of participants whose waist circumference/body weight changed by $-3 \%$ or lower at each assessment compared to baseline during the treatment term); and the rates of achievement of a $5 \%$ reduction in waist circumference and a $5 \%$ reduction in body weight. Baseline was defined as the beginning of the treatment term. In addition, the percentage and amount of change in subcutaneous fat area, adverse reactions, laboratory tests, and blood pressure were assessed. The percentage of participants who were classified as high risk for metabolic diseases at baseline but were not high risk at week 24 of treatment was also calculated. "High risk" was defined as BMI $\geq 25 \mathrm{~kg} / \mathrm{m}^{2}$ and one or more metabolic disease risk factors, or BMI $\geq 25 \mathrm{~kg} /$ $\mathrm{m}^{2}$ and visceral fat area of $\geq 100 \mathrm{~cm}^{2}$ at baseline. "Metabolic disease risk factor" was defined as meeting any of the following criteria: blood glucose level $\geq 126 \mathrm{mg} / \mathrm{dL}$, HbA1c $\geq 6.5 \%$, lowdensity lipoprotein (LDL)-cholesterol $\geq$ $140 \mathrm{mg} / \mathrm{dL}$, high-density lipoprotein (HDL)-cholesterol $<40 \mathrm{mg} / \mathrm{dL}$, triglycerides $\geq 150 \mathrm{mg} /$ $\mathrm{dL}$, systolic blood pressure $\geq 140 \mathrm{mmHg}$, or diastolic blood pressure $\geq 90 \mathrm{mmHg}$.

The primary assessment timepoint was considered for the efficacy endpoints, and the percentage and amount of change in subcutaneous fat area were assessed at the last assessment using the last observation carried forward method. The primary assessment timepoint for the improvement rate in high-risk participants was week 24 of treatment. Participants who completed these assessments comprised the full analysis set (FAS), and those who completed assessments of adverse reactions, laboratory tests, and blood pressure comprised the safety analysis set.

\section{Statistical Analysis}

For the analysis of participant baseline characteristics, frequency analyses were performed according to groups and categories. For continuous data, fundamental statistics were calculated. To investigate intergroup equivalency of participants' characteristics, either a contingency table chi-square test or a two-sample $t$ test was used according to the nature of the data. 
The level of significance was 15\% (two-sided). Analyses of covariance were performed using biased participants' characteristics as covariates and the primary endpoints as dependent variables.

A one-sample $t$ test for intragroup comparisons and a two-sample $t$ test for intergroup comparisons were used for the percentage and amount of change in visceral fat area, waist circumference, body weight, BMI, and subcutaneous fat area. The rates of achievement of waist circumference and body weight reduction were calculated according to the group and the degree of reduction $(\leq-5 \%,>-5 \%$ to $\leq-3 \%,>-3 \%$ to $\leq 0 \%$, and $>0 \%)$. For intergroup comparisons, a contingency table chi-square test was used, and for intergroup and intragroup comparisons, two-sided 95\% confidence intervals (CIs) were calculated. The improvement rate in high-risk participants was determined using the chi-square test, and for differences in laboratory values and blood pressure from baseline to each assessment time, a one-sample Wilcoxon test was used. Since analyses other than the primary endpoint are considered, to aim for supporting the results or considering the cause, multiplicity was not considered. The level of significance was 5\% (two-sided). SAS 9.2 and SAS 9.3 were used for all analyses (SAS Institute Inc., Cary, NC, USA).

\section{RESULTS}

\section{Background of the Participants}

Two hundred eligible participants were randomly enrolled into two groups (100 in the orlistat group and 100 in the placebo group) (Fig. 1). Ten of these participants withdrew from treatment (six, orlistat; four, placebo). Of the remaining 190 participants who completed treatment, 32 (12, orlistat; 20 , placebo) withdrew from the study because they did not fulfill the criteria for entering the post-observation term. The remaining 158 participants (82, orlistat; 76, placebo) entered the post-observation term. Two of the 158 participants (one from each group) withdrew from the study during the post-observation term. None of the
200 participants were excluded from efficacy or safety evaluation, and both the FAS and the safety analysis set consisted of all 200 participants.

Baseline demographics of the participants are summarized in Table 1 . The mean \pm SD age was $45.1 \pm 7.4$ years for the orlistat group and $46.8 \pm 7.4$ years for the placebo group. The number of females was $18(18.0 \%)$ in the orlistat group and $21(21.0 \%)$ in the placebo group. Visceral fat area (mean $\pm \mathrm{SD}$ ) was $121.54 \pm 33.90 \mathrm{~cm}^{2}$ in the orlistat group and $133.03 \pm 42.19 \mathrm{~cm}^{2}$ in the placebo group. The percentage of those who had less than $100 \mathrm{~cm}^{2}$ of visceral fat was $25.0 \%$ in the orlistat group and $21.0 \%$ in the placebo group. There were intergroup biases for age, BMI, target for calorie intake reduction, and visceral fat area.

\section{Efficacy}

\section{Efficacy Endpoints}

The percentage change in visceral fat area from baseline to the last assessment [mean \pm standard error (SE)] was $-13.50 \pm 1.52 \%$ in the orlistat group and $-5.45 \pm 1.50 \%$ in the placebo group; at the last assessment, there was a significantly larger change in the orlistat group compared to the placebo group $(p<0.001)$ (Fig. 2). Furthermore, the reduction rate in visceral fat area was significantly larger at each assessment time in the orlistat group compared to the placebo group. The percentage change in waist circumference from baseline to the last assessment was $-2.51 \pm 0.25 \%$ in the orlistat group and $-1.55 \pm 0.26 \%$ in the placebo group. There was a significantly larger change in reduction of waist circumference in the orlistat group compared to the placebo group at the last assessment ( $p=0.010$ ) (Fig. 3). Furthermore, the percentage change in reduction of waist circumference was significantly larger at each assessment time in the orlistat group compared to the placebo group except at week 12 . When biased participants' characteristics were used for adjustment as different covariates or as a single covariant, intergroup differences were as significant as the results of analyses before 


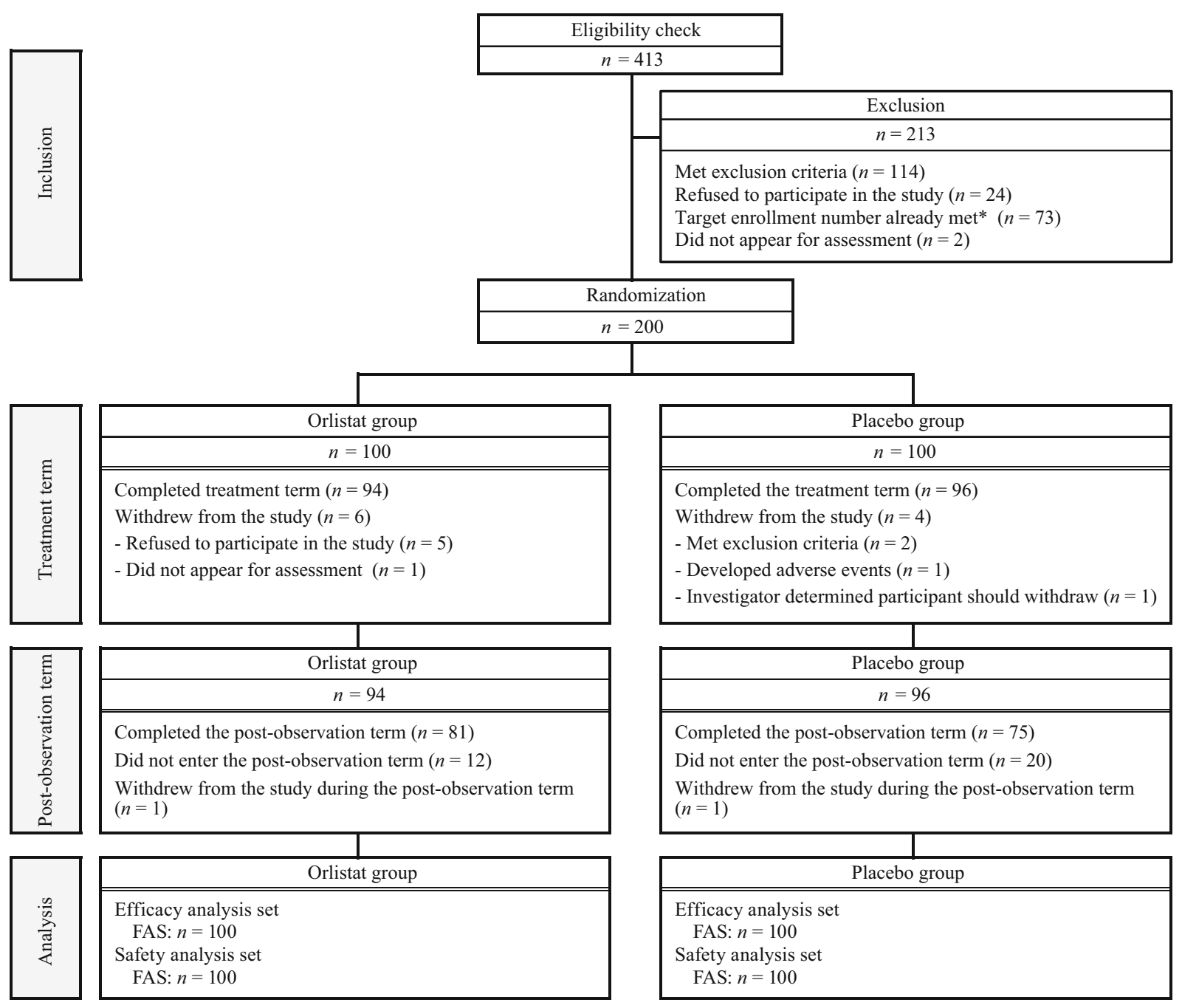

Fig. 1 Disposition of participants. FAS full analysis set. ${ }^{*}$ Participants were withdrawn from the study because a sufficient number of participants had already been recruited

adjustment. No biased participants' characteristics affected the primary endpoints.

The percentage change in body weight from baseline to the last assessment (mean $\pm \mathrm{SE}$ ) was $-2.79 \pm 0.30 \%$ in the orlistat group and $-1.22 \pm 0.28 \%$ in the placebo group. The reduction rate in body weight was significantly larger in the orlistat group compared to the placebo group at the last assessment $(p<0.001)$ (Fig. 4). Furthermore, the reduction rate in body weight was significantly larger in the orlistat group compared to the placebo group at each assessment.

While the percentage changes for visceral fat area, waist circumference, and body weight were comparable in both groups during the observation term, some degree of change in these endpoints was seen in the placebo group and a much greater change was observed in the orlistat group during the treatment term. Although waist circumference and body weight increased during the post-observation term compared to the end of treatment, there was a significantly larger change at post-observation weeks 4,8 , and 12 compared to baseline in both groups (Supplemental Fig. S1).

Secondary endpoints showed similar results, with significantly larger changes in the orlistat group compared to the placebo group (Table 2). The amount of change in visceral fat area from 
Table 1 Participant characteristics at baseline

\begin{tabular}{|c|c|c|c|c|}
\hline Participant characteristics & Statistic & $\begin{array}{l}\text { Orlistat group } \\
n=100\end{array}$ & $\begin{array}{l}\text { Placebo group } \\
n=100\end{array}$ & Test \\
\hline \multirow[t]{7}{*}{ Age (years) } & Mean (SD) & $45.1(7.4)$ & $46.8(7.4)$ & $\chi^{2}=5.521$ \\
\hline & & & & $p=0.137$ \\
\hline & $20-29$ & $2(2.0)$ & $1(1.0)$ & \\
\hline & $30-39$ & $21(21.0)$ & $14(14.0)$ & \\
\hline & $40-49$ & $50(50.0)$ & $43(43.0)$ & \\
\hline & $50-59$ & $27(27.0)$ & $42(42.0)$ & \\
\hline & Min-Max & $27-59$ & $26-59$ & \\
\hline \multicolumn{5}{|l|}{ Gender } \\
\hline \multirow[t]{2}{*}{ Male } & $n(\%)$ & $82(82.0)$ & $79(79.0)$ & $\chi^{2}=0.287$ \\
\hline & & & & $p=0.592$ \\
\hline Female & $n(\%)$ & $18(18.0)$ & $21(21.0)$ & \\
\hline \multirow[t]{3}{*}{ Visceral fat area $\left(\mathrm{cm}^{2}\right)$} & Mean (SD) & $121.54(33.90)$ & $133.03(42.19)$ & $T=-2.115$ \\
\hline & & & & $p=0.036$ \\
\hline & Min-Max & $49.3-245.2$ & $62.2-260.5$ & \\
\hline \multirow[t]{3}{*}{ Waist circumference $(\mathrm{cm})$} & Mean (SD) & $97.64(7.06)$ & $97.40(6.45)$ & $T=0.251$ \\
\hline & & & & $p=0.802$ \\
\hline & Min-Max & $85.5-114.9$ & $85.1-120.0$ & \\
\hline \multirow[t]{3}{*}{ Body weight (kg) } & Mean (SD) & $80.38(10.04)$ & $78.59(8.68)$ & $T=1.347$ \\
\hline & & & & $p=0.180$ \\
\hline & Min-Max & $60.6-105.4$ & $60.7-106.0$ & \\
\hline \multirow[t]{2}{*}{ Height $(\mathrm{cm})$} & Mean (SD) & $170.98(7.12)$ & $170.06(7.49)$ & \\
\hline & Min-Max & $150.5-187.4$ & $150.1-189.0$ & \\
\hline \multirow[t]{7}{*}{ BMI $\left(\mathrm{kg} / \mathrm{m}^{2}\right)$} & Mean (SD) & $27.47(2.75)$ & $27.11(2.50)$ & $\chi^{2}=6.690$ \\
\hline & & & & $p=0.082$ \\
\hline & $22.1-24.9$ & $22(22.0)$ & $19(19.0)$ & \\
\hline & $25.0-29.9$ & $54(54.0)$ & $66(66.0)$ & \\
\hline & $30.0-34.9$ & $24(24.0)$ & $13(13.0)$ & \\
\hline & Unknown & $0(0.0)$ & $2(2.0)$ & \\
\hline & Min-Max & $23.0-33.6$ & $22.6-33.9$ & \\
\hline
\end{tabular}


Table 1 continued

\begin{tabular}{lllll}
\hline Participant characteristics & Statistic & $\begin{array}{l}\text { Orlistat } \\
\text { group } \boldsymbol{n}=\mathbf{1 0 0}\end{array}$ & $\begin{array}{l}\text { Placebo } \\
\text { group } \boldsymbol{n}=\mathbf{1 0 0}\end{array}$ & $\begin{array}{l}\text { Test } \\
\chi^{2}=6.249 \\
\text { Target for calorie intake reduction (kcal/day) }\end{array}$ \\
\hline & 200 & $1(1.0)$ & $5(5.0)$ & $82(82.0)$ \\
& 300 & $75(75.0)$ & $13(13.0)$ \\
\hline
\end{tabular}

$B M I$ body mass index, $S D$ standard deviation

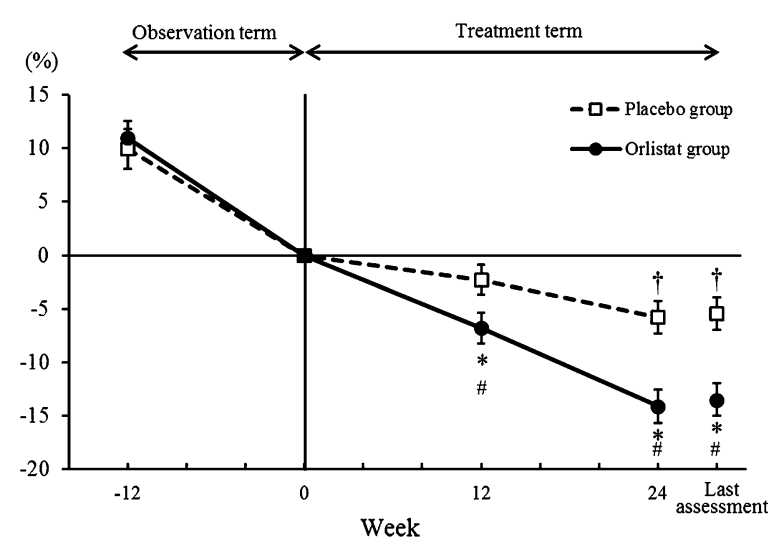

Fig. 2 Percentage change in visceral fat area compared to baseline. Mean \pm SE. $n$ (Last assessment): orlistat, 94; placebo, 97. ${ }^{*}$ Two-sample $t$ test, $p<0.05$ compared to the placebo group; ${ }^{\#, \dagger}$ One-sample $t$ test, $p<0.05$ compared to baseline. Percentage change in visceral fat area was significantly greater in the orlistat group compared to the placebo group at each assessment. SE standard error

baseline to the last assessment (mean \pm SE) was $-15.69 \pm 1.85 \mathrm{~cm}^{2}$ in the orlistat group and $-8.28 \pm 2.01 \mathrm{~cm}^{2}$ in the placebo group ( $p=0.007$, two-sample $t$ test, compared to placebo). Change in waist circumference was $-2.41 \pm 0.24 \mathrm{~cm}$ in the orlistat group and $-1.53 \pm 0.26 \mathrm{~cm}$ in the placebo group $(p=0.016)$, and change in body weight was $-2.21 \pm 0.23 \mathrm{~kg}$ in the orlistat group and $-0.98 \pm 0.23 \mathrm{~kg}$ in the placebo group $(p<0.001)$. The percentage change in BMI was $-2.80 \pm 0.30 \%$ in the orlistat group and $-1.22 \pm 0.28 \%$ in the placebo group $(p<0.001)$, and the amount of change in BMI was $-0.76 \pm 0.08 \mathrm{~kg} / \mathrm{m}^{2}$ in the orlistat group and $-0.34 \pm 0.08 \mathrm{~kg} / \mathrm{m}^{2}$ in the placebo group $(p<0.001)$.

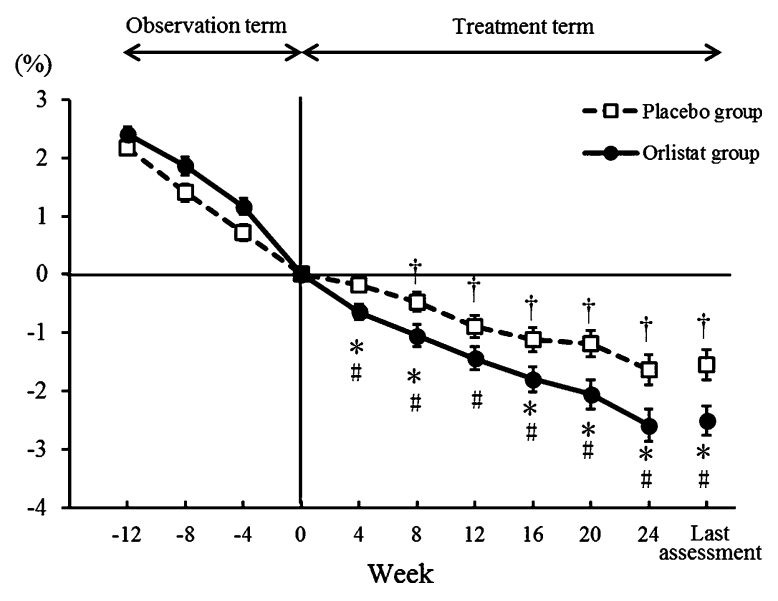

Fig. 3 Percentage change in waist circumference compared to baseline. Mean \pm SE. $n$ (Last assessment): orlistat, 99; placebo, 98. *Two-sample $t$ test, $p<0.05$ compared to the placebo group; ${ }^{\#, \dagger}$ One-sample $t$ test, $p<0.05$ compared to baseline. Percentage change in waist circumference was significantly greater in the orlistat group compared to the placebo group at each assessment except week 12. SE standard error

The rate of achievement of 3\% reduction in waist circumference from baseline to the last assessment was significantly greater in the orlistat group than in the placebo group [38.0\% (95\% CI $28.5-47.5 \%)$ versus $19.0 \%$ (95\% CI $11.3-26.7 \%) ; p=0.003]$. The achievement rate of $5 \%$ waist circumference reduction at the last assessment did not significantly differ between the orlistat group and the placebo group [13.0\% (95\% CI $6.4-19.6 \%)$ versus $10.0 \% \quad(95 \%$ CI $4.1-15.9 \%) ; p=0.506]$.

The achievement rate of $3 \%$ reduction in body weight from baseline to the last assessment was significantly greater in the orlistat 


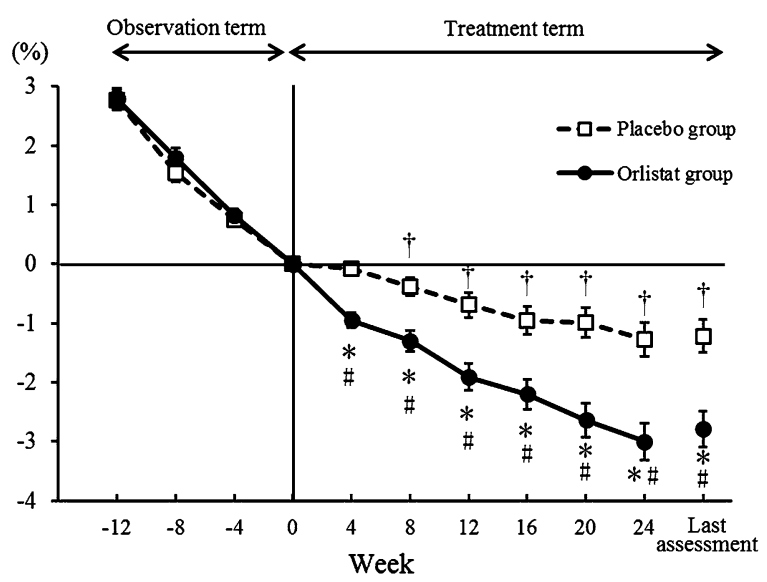

Fig. 4 Percentage change in body weight compared to baseline. Mean \pm SE. $n$ (Last assessment): orlistat, 99; placebo, 98. ${ }^{*}$ Two-sample $t$ test, $p<0.05$ compared to the placebo group; ${ }^{\#, \dagger}$ One-sample $t$ test, $p<0.05$ compared to baseline. Percentage change in body weight was significantly greater in the orlistat group compared to the placebo group at each assessment. SE standard error

group than in the placebo group $[40.0 \%(95 \%$ CI $30.4-49.6 \%)$ versus $19.0 \% \quad(95 \% \quad$ CI $11.3-26.7 \%) ; p=0.001]$. The $5 \%$ body weight reduction achievement rate at the last assessment was significantly greater in the orlistat group than in the placebo group [18.0\% (95\% CI $10.5-25.5 \%)$ versus $8.0 \% \quad(95 \%$ CI $2.7-13.3 \%) ; p=0.036]$.

\section{Clinical Laboratory Tests}

Changes in parameters associated with metabolic diseases, such as total cholesterol, LDL-cholesterol, HDL-cholesterol, and triglycerides, during the treatment term are shown in Fig. 5. The changes in these parameters from baseline to the last assessment (mean $\pm \mathrm{SD}$ ) in the orlistat and placebo groups, respectively, were as follows: total cholesterol, $-4.5 \pm 21.7 \mathrm{mg} / \mathrm{dL}(p=0.007)$ and $5.1 \pm 18.9 \mathrm{mg} / \mathrm{dL} \quad(p=0.003) ; \quad$ LDL-cholesterol, $-6.0 \pm 18.6 \mathrm{mg} / \mathrm{dL} \quad(p<0.001) \quad$ and $2.0 \pm$ $19.6 \mathrm{mg} / \mathrm{dL}(p=0.095) ;$ HDL-cholesterol, $3.4 \pm$ $6.2 \mathrm{mg} / \mathrm{dL} \quad(p<0.001)$ and $4.9 \pm 6.4 \mathrm{mg} / \mathrm{dL}$ $(p<0.001)$; and triglycerides, $-4.6 \pm 37.6 \mathrm{mg} / \mathrm{dL}$ $(p=0.144)$ and $-2.5 \pm 58.4 \mathrm{mg} / \mathrm{dL}(p=0.104)$. Other parameters associated with metabolic diseases, such as blood glucose, HbA1c, systolic blood pressure, and diastolic blood pressure, changed within their normal ranges throughout the study period and did not show any notable changes (Supplemental Fig. S2).

Table 2 Changes in parameters from baseline to the last assessment

\begin{tabular}{|c|c|c|c|c|c|}
\hline Parameter & $\begin{array}{l}\text { Orlistat group } \\
\text { Mean } \pm \text { SE }\end{array}$ & $\begin{array}{l}\text { Placebo group } \\
\text { Mean } \pm \text { SE }\end{array}$ & Parameter & $\begin{array}{l}\text { Orlistat group } \\
\text { Mean } \pm \text { SE }\end{array}$ & $\begin{array}{l}\text { Placebo group } \\
\text { Mean } \pm \text { SE }\end{array}$ \\
\hline $\begin{array}{l}\text { Change in visceral } \\
\text { fat area }\left(\mathrm{cm}^{2}\right)\end{array}$ & $\begin{array}{l}-15.69 \pm 1.85^{\mathrm{a}} \\
(n=94)\end{array}$ & $\begin{array}{l}-8.28 \pm 2.01^{\mathrm{a}} \\
(n=97)\end{array}$ & $\begin{array}{l}\text { Change in waist } \\
\text { circumference }(\mathrm{cm})\end{array}$ & $\begin{array}{l}-2.41 \pm 0.24^{\mathrm{a}} \\
(n=99)\end{array}$ & $\begin{array}{l}-1.53 \pm 0.26^{\mathrm{a}} \\
(n=98)\end{array}$ \\
\hline $\begin{array}{l}\text { Change in body } \\
\text { weight }(\mathrm{kg})\end{array}$ & $\begin{array}{l}-2.21 \pm 0.23^{\mathrm{a}} \\
(n=99)\end{array}$ & $\begin{array}{l}-0.98 \pm 0.23^{\mathrm{a}} \\
(n=98)\end{array}$ & $\begin{array}{l}\text { Percentage change in } \\
\text { BMI (\%) }\end{array}$ & $\begin{array}{l}-2.80 \pm 0.30^{\mathrm{a}} \\
(n=99)\end{array}$ & $\begin{array}{l}-1.22 \pm 0.28^{\mathrm{a}} \\
(n=98)\end{array}$ \\
\hline $\begin{array}{l}\text { Change in BMI } \\
\left(\mathrm{kg} / \mathrm{m}^{2}\right)\end{array}$ & $\begin{array}{l}-0.76 \pm 0.08^{a} \\
(n=99)\end{array}$ & $\begin{array}{l}-0.34 \pm 0.08^{\mathrm{a}} \\
(n=98)\end{array}$ & $\begin{array}{l}\text { Percentage change in } \\
\text { subcutaneous fat area } \\
(\%)\end{array}$ & $\begin{array}{l}-7.53 \pm 1.01^{\mathrm{a}} \\
(n=94)\end{array}$ & $\begin{array}{l}-3.79 \pm 0.88^{\mathrm{a}} \\
(n=97)\end{array}$ \\
\hline $\begin{array}{l}\text { Change in } \\
\text { subcutaneous fat } \\
\text { area }\left(\mathrm{cm}^{2}\right)\end{array}$ & $\begin{array}{l}-17.75 \pm 2.40^{a} \\
(n=94)\end{array}$ & $\begin{array}{l}-9.73 \pm 2.36^{\mathrm{a}} \\
(n=97)\end{array}$ & & & \\
\hline
\end{tabular}

The amount of change in visceral fat area, waist circumference, and body weight as well as the percentage change and amount of change in BMI and subcutaneous fat area were significantly reduced at the last assessment compared to baseline in the orlistat group versus placebo group $B M I$ body mass index, $S E$ standard error

${ }^{a}$ One-sample $t$ test, $p<0.05$ compared to baseline 
(a) Total cholesterol

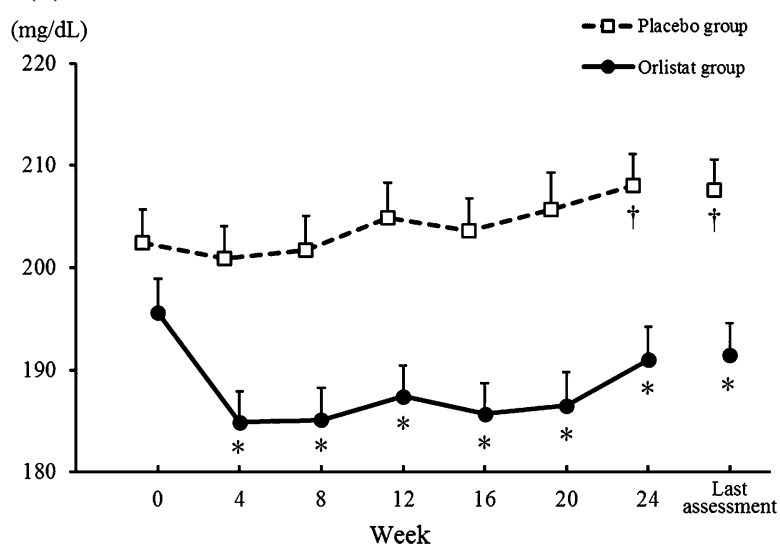

(c) HDL-cholesterol

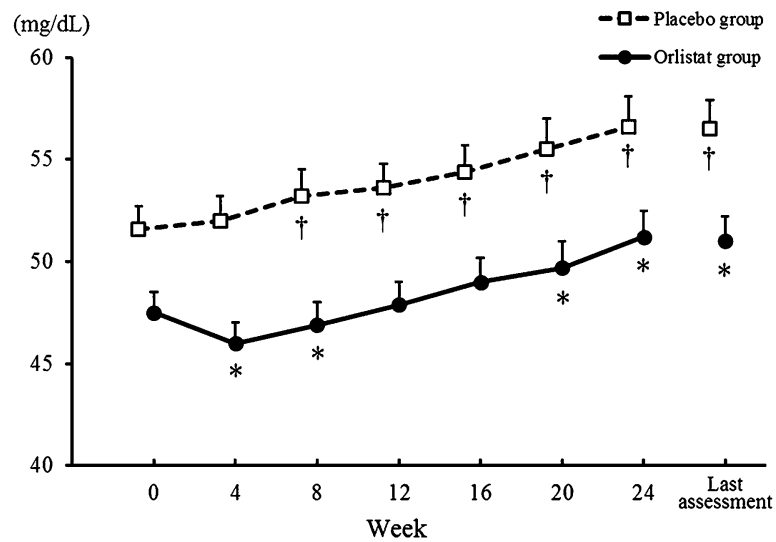

Fig. 5 Changes in lipid parameters. Mean \pm SD. $n$ (Last assessment): a-d orlistat, 100; placebo, $100 .{ }^{*},{ }^{\dagger}$ One-sample Wilcoxon test, $p<0.05$ compared to baseline. In the orlistat group, total cholesterol and LDL-cholesterol were reduced during the treatment term and were significantly

\section{Other Efficacy Analyses}

The improvement rate in high-risk participants at week 24 of treatment was significantly greater in the orlistat group than in the placebo group (32.8\% versus $16.2 \% ; p=0.026$; Table 3$)$.

\section{Safety and Tolerability}

Of the 100 participants in the orlistat group, 82 episodes of adverse reactions were observed in 45 participants $(45.0 \%)$ and 14 episodes were observed in $11(11.0 \%)$ of the 100 participants in the placebo group. In the orlistat group, adverse reactions included defecation-related symptoms that likely resulted from the (b) LDL-cholesterol

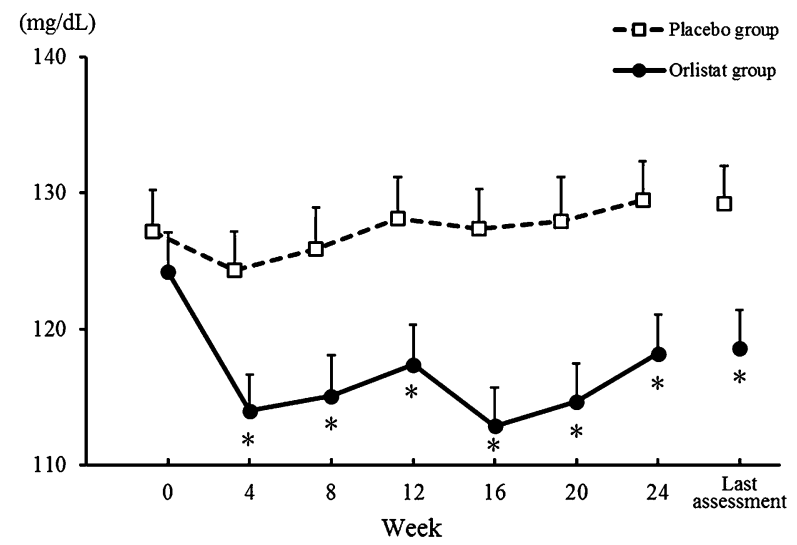

(d) Triglycerides

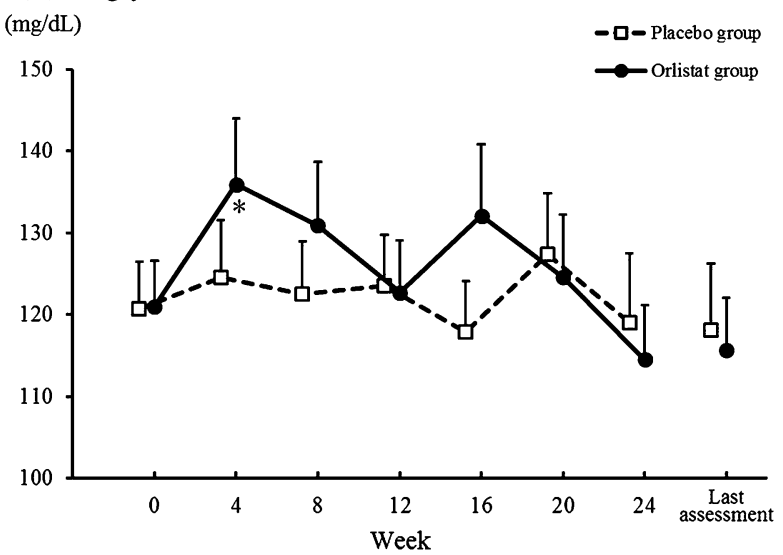

reduced at each assessment compared to baseline. Other parameters stably changed within their normal ranges during the treatment term. HDL high-density lipoprotein, LDL low-density lipoprotein, SD standard deviation

pharmacological effects of orlistat, including oily spotting and flatus with discharge [77 episodes in 41 participants (41.0\%)]; liver function test abnormal [two episodes in two participants (2.0\%)]; malaise [one episode in one participant (1.0\%)]; ureterolithiasis [one episode in one participant (1.0\%)]; and pityriasis rosea [one episode in one participant (1.0\%)]. Participants in the placebo group reported defecation-related symptoms including oily spotting and flatus with stool [13 episodes in 10 participants (10.0\%)] and skin odour abnormal [one episode in one participant (1.0\%)]. In the orlistat group, 78 episodes of adverse reactions in 44 participants $(44.0 \%)$ were of mild severity, and four 
Table 3 Improvement rate in high-risk participants

\begin{tabular}{|c|c|c|c|c|c|c|}
\hline Group & $\begin{array}{l}\text { High-risk } \rightarrow \text { non- } \\
\text { high-risk }\end{array}$ & $\begin{array}{l}\text { Unchanged } \\
\text { (high-risk) }\end{array}$ & $\begin{array}{l}\text { Non- } \\
\text { assessable }\end{array}$ & Total & $\begin{array}{l}\text { Improvement rate } \\
\text { non-high-risk }\end{array}$ & Chi-square test \\
\hline $\begin{array}{r}\text { Orlistat } \\
\text { group }\end{array}$ & $21(32.8)$ & $40(62.5)$ & $3(4.7)$ & 64 & $\begin{array}{l}21 / 64(32.8) \\
\quad[21.3-44.3]\end{array}$ & $\begin{array}{r}\chi^{2}=4.968 \\
p=0.026\end{array}$ \\
\hline $\begin{array}{r}\text { Placebo } \\
\text { group }\end{array}$ & $11(16.2)$ & $56(82.4)$ & $1(1.5)$ & 68 & $\begin{array}{r}11 / 68(16.2) \\
{[7.4-24.9]}\end{array}$ & $(16.6)[2.2-31.1]$ \\
\hline
\end{tabular}

Improvement rate values are presented as $n /$ Total ( $\%)$ ( $95 \%$ confidence interval)

High risk: $\mathrm{BMI} \geq 25 \mathrm{~kg} / \mathrm{m}^{2}+$ one or more metabolic disease risk factors ${ }^{\mathrm{a}}$ or $\mathrm{BMI} \geq 25 \mathrm{~kg} / \mathrm{m}^{2}+\mathrm{visceral}$ fat area $\geq 100 \mathrm{~cm}^{2}$

The improvement rate in high-risk participants at week 24 of treatment was significantly greater in the orlistat group than in the placebo group

$B M I$ body mass index, $H b A 1 c$, glycated hemoglobin, $H D L$ high-density lipoprotein, $L D L$ low-density lipoprotein

${ }^{a}$ Metabolic disease risk factors-blood glucose: fasting blood glucose $\geq 126 \mathrm{mg} / \mathrm{dL}$ or HbA1c $\geq 6.5 \%$. Lipid: LDLcholesterol $\geq 140 \mathrm{mg} / \mathrm{dL}$ or HDL-cholesterol $<40 \mathrm{mg} / \mathrm{dL}$ or triglycerides $\geq 150 \mathrm{mg} / \mathrm{dL}$. Blood pressure: systolic blood pressure $\geq 140 \mathrm{mmHg}$ or diastolic blood pressure $\geq 90 \mathrm{mmHg}$

episodes in three participants (3.0\%) were moderate; no severe adverse reactions were observed. In the placebo group, 14 mild episodes of adverse reactions were observed in 11 participants $(11.0 \%)$, with no instances of moderate or severe adverse reactions.

The predominant adverse reactions were defecation-related symptoms caused by fecal oil excretion, which likely resulted from the pharmacological effects of orlistat. Most episodes were mild with transient and subjective symptoms, and no serious or severe episodes were observed. No episodes required medical treatment, and no participants were advised by the physician to withdraw from treatment.

No adverse reactions related to fat-soluble vitamin deficiencies were observed, and no participants required treatment or vitamin supplementation. Levels of vitamin A, D, and $\mathrm{E}$ remained steady during the treatment term and did not show notable changes (Supplemental Fig. S2).

\section{DISCUSSION}

This study was conducted to evaluate the efficacy and safety of orlistat $(60 \mathrm{mg}$ administered three times daily for 24 weeks) in Japanese participants without metabolic diseases despite excessive visceral fat accumulation. This is the first report to investigate the effect of orlistat in reducing visceral fat in a Japanese population. Moreover, in addition to the reduction in visceral fat area, waist circumference, body weight, BMI, and subcutaneous fat area were reduced significantly compared to the placebo group. The majority of adverse reactions were mild defecation-related symptoms caused by fecal oil excretion due to the pharmacological effects of lipase inhibitors.

In the USA and Europe, a 120-mg formulation of orlistat is available as a prescription drug (Xenical $^{\circledR}$, F. Hoffman-LaRoche, Ltd., Basel, Switzerland), and a 60-mg formulation as a nonprescription drug (Alli ${ }^{\circledR}$, GlaxoSmithKline, Brentford, UK). Many studies have been conducted to evaluate the efficacy and safety of orlistat in patients with severe obesity in these countries, and significant body weight changes were observed as well in a previous study (orlistat was administered three times daily for 104 weeks at a dose of $60 \mathrm{mg}$ to severely obese patients with BMI between 30 and $44 \mathrm{~kg} / \mathrm{m}^{2}$ ) [17].

Other anti-obesity drugs, such as lorcaserin and the combination of bupropion and naltrexone, have recently been approved in some countries. Significant body weight reductions have been demonstrated in clinical studies for lorcaserin (in obese patients with BMI 30-45 kg/ 
$\mathrm{m}^{2}$ or those with $\mathrm{BMI} \geq 27 \mathrm{~kg} / \mathrm{m}^{2}$ with hypertension and/or dyslipidemia) [18] and the combination of bupropion and naltrexone (in obese patients with $\mathrm{BMI} \geq 30 \mathrm{~kg} / \mathrm{m}^{2}$ or those with $\mathrm{BMI} \geq 27 \mathrm{~kg} / \mathrm{m}^{2}$ with hypertension and/or dyslipidemia) [19]. Furthermore, the frequency of headache, nausea, and dizziness was significantly higher in the lorcaserin group compared with the placebo group, while the combination of bupropion and naltrexone caused nausea, headache, constipation, dizziness, and dry mouth [20]. In addition, many episodes of neuropsychiatric disorders have been reported with these two drugs, whereas the predominant adverse reaction of orlistat is defecation-related symptoms [20].

During the observation term of this study, reductions in visceral fat area, waist circumference, and body weight were comparable between the orlistat group and the placebo group. This may be attributed to dietary improvements and the effects of interventions during the observation term. During the treatment term, visceral fat area, waist circumference, and body weight were significantly and consistently reduced in the orlistat group, and these parameters were minimally reduced in the placebo group. Similar findings were observed in BMI and subcutaneous fat area, with reduction rates of these parameters being significantly larger in the orlistat group than in the placebo group. Taken together, these results suggest that the efficacy of orlistat is greater than the therapeutic effects of dietary improvements alone, and can successfully complement adjustments to diet and exercise. Orlistat may be a useful option to prevent metabolic diseases caused by obesity and could potentially play an important role in early obesity treatment. However, the changes observed in waist circumference and body weight during the post-observation term of this study suggest that lifestyle improvement remains crucial after the completion of treatment with orlistat.

Orlistat is believed to suppress triglyceride absorption and thereby reduce visceral fat and body weight. Reduction in waist circumference can be attributed to subsequent abdominal fat reduction. Because orlistat reduces food-derived fat absorption by $25-30 \%$ [15], orlistat is considered to reduce serum lipid levels in the blood. In this study, total cholesterol and LDLcholesterol were significantly reduced in the orlistat group during the treatment term compared to baseline. Although HDL-cholesterol was significantly increased at the last assessment, no notable improvements were observed. Triglyceride levels fluctuated within the normal range without notable changes. In a metaanalysis by $\mathrm{Hu}$ et al., participants receiving a low-carbohydrate diet showed a greater increase in HDL-cholesterol and reduction in triglycerides compared to those receiving a low-fat diet, but experienced less reduction in LDL-cholesterol compared with those receiving a lowfat diet [21]. Orlistat, then, likely did not affect HDL-cholesterol or triglycerides, which are more likely to be affected by carbohydrate intake rather than fat intake, as orlistat's mechanism of action inhibits the absorption of food-derived fat.

Blood glucose, HbA1c, systolic blood pressure, and diastolic blood pressure also varied within their normal ranges. The direct effects of orlistat on blood glucose level and blood pressure remain unclear; this study involved participants with normal blood glucose levels and blood pressure. However, it may be reasonable to expect that orlistat can prevent aggravation of blood glucose levels and blood pressure imbalances in the long term.

Although no participants in this study had metabolic diseases, in addition to reductions in visceral fat area and waist circumference, the improvement rate in high-risk participants was significantly greater in the orlistat group compared to the placebo group, suggesting that reducing visceral fat helps mitigate the risk of metabolic diseases caused by obesity.

As mentioned above, this study is the first to report reductions in visceral fat and improvements in other parameters in response to orlistat administered for 24 weeks at a dose of $60 \mathrm{mg}$ in Japanese subjects. Most adverse reactions were mild, and defecation-related symptoms caused by fecal oil excretion, enhanced by the pharmacological effects of orlistat, accounted for the majority of adverse reactions. Because these symptoms were subjective and were cured 
or relieved during or after treatment with the study drug, these adverse reactions were considered to be reversible and tolerable.

Waist circumference was used to select participants, and as a result, those with a visceral fat area of less than $100 \mathrm{~cm}^{2}$ were included in this study (as in other studies) [6]. Notably, not only was visceral fat reduced in these participants but waist circumference, which serves as an alternative index for visceral fat area, was also reduced.

The results of this study suggest that orlistat $60 \mathrm{mg}$ is effective for improving obesity as a complement to diet and exercise, thereby mitigating the health risks associated with metabolic diseases caused by obesity. We expect that orlistat will become a useful drug to reduce visceral fat accumulation.

The study has a few limitations. The treatment term of this study was relatively short, and the sample size might have been too small to evaluate the development of metabolic diseases in obese patients. Additionally, the study included only Japanese patients and inter-racial differences were not considered.

\section{CONCLUSION}

In this study, orlistat $60 \mathrm{mg}$ was administered three times daily for 24 weeks to participants in Japan at risk for metabolic diseases due to excessive visceral fat accumulation. Significant reductions in visceral fat area and waist circumference were observed with a tolerable safety profile, suggesting that the use of orlistat can reduce visceral fat accumulation and mitigate the risk of developing metabolic diseases caused by obesity.

\section{ACKNOWLEDGEMENTS}

The authors would like to thank the participants, investigators, and other staff members for their invaluable contributions to the study. We would like to acknowledge Dr. Yutaka Kimura (Kansai Medical University) and Dr. Ichiro Tatsuno (Sakura Medical Center, Toho University) for support and implementation of this study. We would like to acknowledge Dr. Norio Tada (Jikei University Kashiwa Hospital) for interpretation of the study results.

Funding. Funding for this study, manuscript preparation, article processing charges and the open access fee was provided by Taisho Pharmaceutical Co., Ltd. (Tokyo, Japan). All authors have sufficient access to all data of this study and are responsible for the integrity of the data and accuracy of the data analysis.

Editorial Assistance. Editorial assistance in the preparation of this article was provided by Pearl Gomes of Cactus Communications (Tokyo, Japan). Support for this assistance was funded by Taisho Pharmaceutical Co., Ltd. (Tokyo, Japan).

Authorship. All named authors meet the International Committee of Medical Journal Editors (ICMJE) criteria for authorship of this article, take responsibility for the integrity of the work as a whole, and have given their approval for this version to be published.

Disclosures. Dr. Kohji Shirai received compensation from Taisho Pharmaceutical Co., Ltd. (Tokyo, Japan) for contribution to manuscript preparation and submission. Toru Fujita is an employee of Taisho Pharmaceutical Co., Ltd. (Tokyo, Japan). Yuka Fujii is an employee of Taisho Pharmaceutical Co., Ltd. (Tokyo, Japan). Masatsugu Shimomasuda is an employee of Taisho Pharmaceutical Co., Ltd. (Tokyo, Japan). Michitaka Tanaka is an employee of Taisho Pharmaceutical Co., Ltd. (Tokyo, Japan). Soichi Sakai is an employee of Taisho Pharmaceutical Co., Ltd. (Tokyo, Japan). Yoshishige Samukawa is an employee of Taisho Pharmaceutical Co., Ltd. (Tokyo, Japan).

Compliance with Ethics Guidelines. The study was implemented in accordance with Good Clinical Practice guidelines and the ethical principles of the Helsinki Declaration of 1964, as revised in 2013, concerning human and animal rights, and Springer's policy concerning informed consent has been followed. This study was approved by the institutional 
review board of each participating institution. After receiving a sufficient explanation and achieving a full understanding of the study, all potential participants provided written consent to voluntarily participate in the study.

Data Availability. The datasets generated during and/or analyzed during the current study are not publicly available because of confidentiality reasons but are available from the corresponding author on reasonable request.

Open Access. This article is distributed under the terms of the Creative Commons Attribution-NonCommercial 4.0 International License (http://creativecommons.org/licenses/ by-nc/4.0/), which permits any noncommercial use, distribution, and reproduction in any medium, provided you give appropriate credit to the original author(s) and the source, provide a link to the Creative Commons license, and indicate if changes were made.

\section{REFERENCES}

1. Miyazaki S. From obesity and obesity disease to metabolic syndrome. J Ther. 2008;90:1650-4.

2. Japan Society for the Study of Obesity. Guidelines for the management of obesity disease. Life Science Publishing Co.; 2016. p. 1-132.

3. Sassi F. In: Sassi F, editor. Obesity and the economics of prevention: Fit not fat. Cheltenham: Edward Elgar; 2010.

4. Yumuk V, Frühbeck G, Oppert JM, Woodward E, Toplak H. An EASO position statement on multidisciplinary obesity management in adults. Obes Facts. 2014;7(2):96-101.

5. NIH. Clinical guidelines on the identification, evaluation, and treatment of overweight and obesity in adults: the evidence report. 1998. https:// www.healthypeople.gov/2020/tools-resources/evid ence-based-resource/clinical-guidelines-on-the-iden tification-evaluation. Accessed 10 Apr 2018.

6. Hiuge-Shimizu A, Kishida K, Funahashi T, et al. Absolute value of visceral fat area measured on computed tomography scans and obesity-related cardiovascular risk factors in large-scale Japanese general population (the VACATION-J study). Ann Med. 2012;44:82-92.

7. Kato A, Muramoto A, Matsushita M, Tsushita K. The relationship between the change of visceral fat area using dual impedance method and the improvement of medical data related to obesity: evaluation of Japanese men in their 20's and 30's with obesity who have gone through a life style intervention program. J Jpn Soc Study Obes. 2016;22:117-23.

8. Umebayashi A, Kanesada Y, Nishikawa $\mathrm{H}$, et al. Relationships between risk factors for metabolic syndrome and visceral fat analyzed by age groups. J Jpn Mibyo Syst Assoc. 2015;21:1-6.

9. Okauchi Y, Nishizawa H, Funahashi T, et al. Reduction of visceral fat is associated with decrease in the number of metabolic risk in Japanese men. Diabetes Care. 2007;30:2392-4.

10. Look AHEAD Research group, Pi-Sunyer X, Blackburn $\mathrm{G}$, et al. Reduction in weight and cardiovascular disease risk factors in individuals with type 2 diabetes: one-year results of the look AHEAD trial. Diabetes Care. 2007;30:1374-83.

11. Yamaha Health Insurance Society. The second-term implementation plan of specific health checkup, etc. (fiscal year 2013-2017). https://www.yama hakenpo.or.jp/wp-content/themes/kenpo/file/no913.pdf. Accessed 10 Apr 2018.

12. Mutual aid association for Tokyo metropolitan government officials, data health plans. March 31, 2015. https://www.kyosai.metro.tokyo.jp/info/detail/ data/info_data/20150330/datahealth.pdf. Accessed 10 Apr 2018.

13. Muramoto A, Matsushita M, Kato A, et al. Three percent weight reduction is the minimum requirement to improve health hazards in obese and overweight people in Japan. Obes Res Clin Pract. 2014;8:e466-75.

14. Hiratani M, Nakamura S, Nakanishi S, Kihira E. Effects of specific health instruction: four years hence. J Rural Med. 2015;64:34-40.

15. Smith SR, Stenlof KS, Greenway FL, et al. Orlistat $60 \mathrm{mg}$ reduces visceral adipose tissue: a 24-week randomized, placebo-controlled, multicenter trial. Obesity. 2011;19:1796-803.

16. Matsuzawa Y, Kotani K, Tokunaga K. Ideal body weight with the lowest morbidity. J Jpn Soc Study Obes. 1998;4:65-9.

17. Hauptman J, Lucas C, Boldrin MN, Collins H, Segal KR. Orlistat in the long-term treatment of obesity in primary care settings. Arch Fam Med. 2000;9:160-7. 
18. Highlights of prescribing information (BELVIQ). https://www.belviq.com/-/media/Files/BelviqConso lidation/PDF/Belviq_Prescribing_information-pdf. Accessed 10 Apr 2018.

19. Highlights of prescribing information (Contrave). https://www.accessdata.fda.gov/drugsatfda_docs/ label/2017/200063s009lbl.pdf. Accessed 10 Apr 2018.
20. Ueno H, Nakazato M. The prospects of novel antiobesity drugs. J Jpn Soc Intern Med. 2014;103:753-9.

21. $\mathrm{Hu} \mathrm{T}$, Mills KT, Yao L, et al. Effects of low-carbohydrate diets versus low-fat diets on metabolic risk factors: a meta-analysis of randomized controlled clinical trials. Am J Epidemiol. 2012;176:S44-54. 